\title{
Relato de Experiência: Comunidade de Inteligência Artificial para Mulheres
}

\author{
Poliana Nascimento Ferreira ${ }^{1,2}$, Laura Damaceno de Almeida ${ }^{1,3}$, \\ Andressa da Silva Siqueira ${ }^{1,4}$, Beatriz Pereira ${ }^{1}$
}

${ }^{1}$ Comunidade AI Girls

${ }^{2}$ Centro de Matemática, Computação e Cognição, Universidade Federal do ABC Av.dos Estados, 5001 - Bairro S. Terezinha, Santo André, SP, Brasil - CEP: 09210-580

${ }^{3}$ Universidade Anhembi Morumbi R. Jaceru, 247 - Morumbi, São Paulo, SP, Brasil CEP: 04705-000

${ }^{4}$ Departamento de Computação, Instituto Militar de Engenharia Praça Gen. Tibúrcio, 80 - Urca, Rio de Janeiro - RJ, 22290-270

\{poliana.nferreira, siq.andressa, beagomesp\}@gmail.com, laura.da.almeida@hotmail.com

\begin{abstract}
The AI Girls tech community aims to promote female leadership in Artificial Intelligence, given the lack of diversity in the area. In this experience report, AI Girls is presented since its foundation. The organization takes place through areas and structured planning meetings between the coordinators. The actions involve various events and projects, such as reading and mentoring articles, always taking into account the needs of the participants. There are currently more than 4000 women in the community and more than 35 hours of content produced in one year. The feedbacks collected through questionaries and written and verbal testimonies attest to the initiative's success and shape the next steps to be taken.
\end{abstract}

Resumo. A comunidade de tecnologia AI Girls tem o objetivo de promover o protagonismo feminino em Inteligência Artificial, vista a falta de diversidade na área. Neste relato de experiência, é apresentada a AI Girls desde a sua fundação. A organização dá-se através de áreas e reuniões estruturadas de planejamento entre as coordenadoras. As ações envolvem eventos e projetos variados, como clube de leitura e mentoria de artigos, sempre levando em consideração as necessidades das participantes. Atualmente, há mais de 4000 mulheres na comunidade e mais de 35 horas de conteúdo produzido em um ano. Os feedbacks coletados através de pesquisa, depoimentos escritos e verbais atestam o sucesso da iniciativa e moldam os próximos passos.

\section{Introdução}

As comunidades de tecnologia reúnem indivíduos com o objetivo similar de aprender. Com organização informal, são realizados encontros on-line e off-line que promovem um espaço de aprendizagem para todos os atores envolvidos (palestrantes, organizadores e participantes) [Liu et al.. 2012]. Tais encontros, ou meetups, fornecem um espaço no qual a palavra de ordem é compartilhamento, em que a heterogeneidade é valorizada e os diferentes pontos de vista acrescentam dentro do saber coletivo através das teias de relacionamentos [Ferrazza 2020]. 
Cada vez mais os eventos promovidos pelas comunidades tem impacto na qualidade do desenvolvimento tecnológico e pessoal, sendo que no Reino Unido alguns analistas econômicos utilizam dados de tais eventos promovidos para medir a saúde do setor local de tecnologia [Ingram and Drachen 2020]. Em um fenômeno que produz espaços de aprendizagem fora da universidade, o conteúdo é reciclado e atualizado, consonante à fluidez que as novidades na área surgem [Ingram and Drachen 2020].

Observando, entretanto, o panorama geral do público presente em meetups, encontram-se pessoas de diferentes faixas etárias e econômicas [Sander 2005], mas poucas mulheres. Isso é algo preocupante, uma vez que tais eventos refletem o mercado. E de fato, no Brasil, a presença feminina em TI é de apenas 20\% [BRASSCOM 2020].

Levando tais dados em consideração, diversas iniciativas foram criadas com o intuito de promover o protagonismo feminino em tecnologia. São as comunidades femininas de tecnologia brasileiras, as quais podemos citar Pyladies, $R$ Ladies e InspirAda. De acordo com Vieira et al. (2017), as mulheres participantes nas comunidades se sentem mais confortáveis em participar quando estão entre mulheres. Peres e Gomes (2020) apresentaram indícios da contribuição das comunidades femininas para o aumento de adesão da área de tecnologia, destacando também a importância do empoderamento para tal consolidação. Santiago e de Andrade (2018) destacam vivências positivas de estudantes, mentores e embaixadores na comunidade. Já Souza et al. (2017) mostram impactos preliminares da rede construída pelas mulheres no ecossistema local, promovendo diversidade e inclusão no mercado de tecnologia.

Mostra-se então, um espaço em que há necessidade e grandes benefícios para a criação de comunidades femininas de tecnologia. Este trabalho apresenta, através de relato de experiência, a comunidade AI Girls ${ }^{12}$ desde a sua fundação, destacando as contribuições para as participantes e a forma de organização da comunidade. Espera-se, com isso, ressaltar a importância de tais iniciativas, auxiliar mulheres na criação de suas próprias redes em suas cidades e também integrar mais o espaço acadêmico ao das comunidades de tecnologia.

O trabalho está organizado da seguinte forma: na seção 2 é mostrada a motivação presente na criação da comunidade. Na seção 3 são apresentados a organização da comunidade e os diversos papeis representados por suas coordenadoras, além de suas iniciativas. A seção 4 mostra o crescimento da comunidade e seus resultados obtidos enquanto a seção 5 apresenta as considerações finais

\section{Motivação}

$\mathrm{Na}$ área de foco da comunidade, a Inteligência Artificial (IA), a falta de diversidade de gênero cria o risco de os sistemas de IA perpetuarem a desigualdade estrutural [Stathoulopoulos and Mateos-Garcia 2019]. A edição de 2018 do The Global Gender Gap Report [World Economic Forum 2018] trouxe, com destaque, diversas considerações sobre a presença feminina na área. Na pesquisa, é apresentado um gap significativo, com apenas $22 \%$ de mulheres na área, sendo este número no Brasil de apenas $14 \%$. Outro dado interessante é que a crescente busca por conhecimentos em IA não apresenta diferenças

\footnotetext{
${ }^{1}$ https://aigirlsbr.github.io/

${ }^{2}$ https://linktr.ee/Ai_girls
} 
percentuais entre homens e mulheres, indicando uma significativa perda de oportunidade na contração de pessoal em um domínio profissional onde já há oferta insuficiente de mão de obra qualificada. Em 2020 os números aumentaram, mas ainda não o suficiente, com 26\% de participação mundial e 18\% no Brasil [World Economic Forum 2020].

Vê-se então um problema em que há igual interesse de mulheres pela área, mas o mercado não reflete isso. A comunidade tem como motivação justamente atacar esse problema, promovendo espaços confortáveis para as mulheres se desenvolverem, mostrando que a área de IA também é o lugar delas e criando um pool de profissionais mulheres qualificadas.

\section{Organização da Comunidade}

A AI Girls foi estabelecida em janeiro de 2020, quando o grupo da organização percebeu uma dor em comum de muitas mulheres com interesse na área de IA: falta de incentivo para entrar na área, falta de pertencimento e falta de um espaço confortável para troca de conhecimentos e experiências. Essa percepção foi derivada da participação em outras comunidade sobre o tema de Inteligência Artificial. Não havia muitas mulheres que se manifestavam com frequência nos grupos ou palestravam. Investigando mais a fundo, conversando com algumas meninas e com inspiração em outras comunidades femininas de tecnologia, surgiu a ideia de criar esse espaço específico e independente para Elas.

A comunidade AI Girls, então, tem como missão promover o protagonismo feminino nas áreas de inteligência artificial e ciência de dados. Incentiva abrindo espaço para mulheres palestrarem, conduzirem workshops e escreverem artigos e qualifica através de meetups, workshops, mentorias e networking gratuitos. A primeira etapa no processo de criação da comunidade foi a divisão de áreas e divisão de responsabilidades entre as coordenadoras. Cada área possui um manual, no qual é descrito o que é feito e como é feito, possibilitando a fácil integração de novas coordenadoras e mantendo uma relação de transparência na organização. Além disso, outras responsabilidades, surgidas a partir da necessidade com o decorrer do tempo, compreendem a manutenção do site, contato com patrocinadores e ideação de novos projetos. Atualmente a organização, composta por seis mulheres, é dividida em quatro grandes áreas:

- Rede Social - gerenciamento das redes sociais.

- Eventos - criação e acompanhamento dos eventos, meetups e workshops.

- Artes - responsável pela identidade visual e artes usadas para divulgação.

- Conteúdo - curadoria e escrita dos conteúdos postados no blog.

O local principal de tomadas de decisão na comunidade são reuniões mensais realizadas pela plataforma Google Meet. Com duração média de duas horas e realizadas no primeiro sábado de cada mês, são definidas atividades e prioridades para o mês subsequente, apresentadas novas ideias e são feitos resumos do que foi realizado e progressos do mês anterior. É escrita uma ata sobre o que foi discutido, a qual contém do estado de cada um dos projetos, sua prioridade (Stand By, Baixa, Média, Alta, Muito Alta e Feito) e a responsável por ele. Além disso, há um grupo no Telegram para facilitar a comunicação, no qual trocamos ideias sobre assuntos mais urgentes do mês atual.

As iniciativas novas surgem a partir das necessidades das participantes da comunidade. Há constante troca de experiências e observação da movimentação na comunidade, evidenciando quais são os temas mais pesquisados ou debatidos. Estes temas são 
abordados nas reuniões mensais para que sejam aludidos no planejamento de conteúdo e entregas. Para início de um projeto, a coordenadora idealizadora redige um documento constando o objetivo e fases de implementação do novo projeto, colocando todas a par e observando se aquilo é de fato factível. Quando esse projeto é consolidado, o planejamento vira um manual.

O ano de 2020 foi um ano de estabelecimento da comunidade, seus objetivos e projetos. O primeiro passo foi a criação de um grupo no Telegram, criando assim uma rede de apoio entre as participantes. Após o grupo, foram feitas as redes sociais do Instagram ${ }^{3}$, Facebook $^{4}$ e Linkedin ${ }^{5}$. E, posteriormente, também o Github ${ }^{6}$, o Youtube ${ }^{7}$ e o Dev.to ${ }^{8}$ (blog com conteúdos).

\subsection{Iniciativas e Projetos}

Os projetos da comunidade são pensados para veicular da melhor maneira o conhecimento e mulheres inspiradoras na área e também para promover um espaço seguro de compartilhamento para as participantes. Eles foram surgindo aos poucos, sendo que, atualmente, tem-se as seguintes iniciativas:

Eventos: pilar central da comunidade, a realização de meetups e workshops contribui para oferecer um espaço para as mulheres palestrarem e serem ouvidas, além de compartilhar conteúdo de qualidade com a comunidade. Os meetups presenciais ocorrem em parceria com empresas, as quais fornecem espaço para a realização. Em 06 de fevereiro de 2020, a AI Girls teve seu primeiro evento cujo tópico discutido foi o papel da mulher na área de ciência de dados, a importância da diversidade nos times de Analytics e introdução a alguns tópicos da IA: machine learning e visão computacional. A partir do mês de março de 2020, devido à pandemia do CoVID-19, a comunidade decidiu realizar os eventos de forma on-line, voltando a ter seu primeiro evento on-line realizado no dia 04 de abril sobre os problemas sexistas e separatistas que alguns sistemas de inteligência artificial podem ter e como podemos evitar que isso aconteça.

Vale destacar alguns eventos específicos que aconteceram, tais como o Especial de Dados, no mês de maio, a participação no Female Tech Leaders Summit, em maio, e o evento de aniversário de um ano da comunidade, em fevereiro de 2021. O aniversário contou com três dias seguidos de conteúdo e trilhas diferentes (Carreira e Representatividade, Dados, IA).

Dona Cientista: o projeto Dona Cientista tem como objetivo principal mostrar a história de várias mulheres que contribuíram significativamente para a tecnologia ao longo do tempo, já foi contada a história de dezessete mulheres pela comunidade. Para o desenvolvimento deste projeto, é feita uma extensa pesquisa sobre essas mulheres, suas histórias, seus desafios e também suas realizações. A partir dai é escrito um texto pequeno e colocado nas redes sociais com suas fotos e/ou gravuras. Esse projeto ajuda a mostrar que as mulheres sempre tiveram no meio acadêmico e empresarial, fazendo novas descobertas e ajudando a moldar o mundo como conhecemos hoje.

\footnotetext{
${ }^{3}$ https://www.instagram.com/aigirlsbrasil

${ }^{4}$ https://www.facebook.com/aigirlsbr/

${ }^{5} \mathrm{https} / / /$ www.linkedin.com/company/ai-girls/

${ }^{6}$ https://github.com/aigirlsbr

${ }^{7}$ https://www.youtube.com/c/AIGirlsComunidade

${ }^{8}$ https://dev.to/aigirlsbr
} 
Wonderful Woman: o projeto Wonderful Woman é um projeto em que são convidadas mulheres referências na área para falarem sobre suas trajetórias profissionais e conectando assim mulheres que já estão na área com as iniciantes.

Clube de Leitura: tem o objetivo de fazer as meninas adquirirem conhecimento, diversificarem o repertório e familiarizar algumas mulheres sobre inteligência artificial. No dia 22 de Setembro de 2020, o clube de leitura da comunidade fez seu primeiro encontro. Foi selecionado um livro introdutório, com conceitos importantes para as pessoas que querem entrar na área: "Inteligência Artificial: Uma Abordagem de Aprendizado de Máquina"[Faceli et al.. 2011].

Projeto Mentoria de Artigos: tem como objetivo ensinar o básico sobre produção de artigos científicos, incluindo modo de pesquisa, ferramentas e regras usadas e assim ter mais mulheres produzindo conteúdos científicos. Este projeto foi lançado no dia 09 de junho de 2020 e ocorre de forma on-line, em que são abertas turmas e a mentora disponibiliza materiais de estudos e realiza chamadas de vídeo para tirar as dúvidas e fazer o acompanhamento dos projetos.

Projeto Café com Rosie: uma iniciativa realizada no blog cujo objetivo é entrevistar as mulheres brasileiras protagonistas da área de IA e ciência de dados e conhecer suas trajetórias, desafios na área, exaltar suas conquistas e qual a opinião das entrevistadas referentes a alguns assuntos polêmicos da área.

AI Girls in English: ocorre de forma on-line e tem objetivo de fazer as meninas da comunidade sentirem-se confortáveis em falar e escrever em inglês. Há um grupo no Telegram para compartilhamento de materiais em inglês e encontros nos quais as meninas revisam a gramática e trocam conhecimentos.

Sobre o grupo de inglês, destaca-se que surgiu espontaneamente através das participantes da comunidade. Elas criaram o grupo e o formato das reuniões sozinhas e as coordenadoras só fazem o papel de apoio e divulgação.

A Tabela 1 apresenta um resumo da linha do tempo referente aos projetos realizados pela comunidade desde a sua criação.

Tabela 1. Linha do Tempo

\begin{tabular}{|c|c|}
\hline \multirow[t]{2}{*}{$01 / 2020$} & Estabelecimento AI Girls \\
\hline & Criação das Redes Sociais \\
\hline \multirow[t]{2}{*}{$02 / 2020$} & 1o Evento Oficial - Presencial \\
\hline & Início projetos: Blog, Dona Cientista, Dica da Semana, Wonderful Woman \\
\hline $04 / 2020$ & 1o Evento On-line - Youtube \\
\hline \multirow[t]{2}{*}{$05 / 2020$} & Café com Rosie \\
\hline & Especial de Dados \\
\hline 06/2020 & Projeto Mentoria de Artigos Científicos \\
\hline $09 / 2020$ & Clube de Leitura \\
\hline $10 / 2020$ & Participação Female Tech Leaders Summit \\
\hline $11 / 2020$ & AI Girls in English \\
\hline $12 / 2020$ & "Férias"da Comunidade \\
\hline $02 / 2021$ & Evento do Aniversário de 1 ano \\
\hline
\end{tabular}




\section{Resultados e Discussões}

Em um ano, a comunidade demonstrou bons resultados, vistos através da presença em redes sociais e dos feedbacks fornecidos durante os eventos realizados.

\subsection{Crescimento da Comunidade}

A comunidade mostrou um grande crescimento, estando presente no Instagram, Facebook, LinkedIn, Youtube e Telegram (Tabela 2). O maior alcance da comunidade segue sendo atráves de avisos no grupo do Telegram, LinkedIn e Instagram, respectivamente. $\mathrm{O}$ crescimento indica o potencial de sucesso dos projetos que estão sendo desenvolvidos e interesse pelo tema.

No Telegram, plataforma com maior interação, são constantemente compartilhadas vagas de empresas, dúvidas, experiências e projetos bem sucedidos. Por dados colhidos entre 22 de fevereiro de 2021 e 07 de março, o horário de mais movimento do grupo é entre 16:00 e 1:00 e em dias da semana. Isso mostra uma predileção por horários após o expediente, mas ainda em dias de trabalho, refletindo o uso do grupo como apoio ao aprendizado e carreira e não apenas recreação.

As informações mostradas na Tabela 2 são respectivamente:

- Número de seguidores - Numero de seguidores em cada rede social

- Alcance - Mostra a quantidade de visualizações do conteúdo por usuário único.

- Impressões - Mostra a quantidade de vezes que seu conteúdo foi visto, sem diferenciar se houve mais de uma visualização por usuário.

- Envolvimento - Mostra a quantidade de interações com seu conteúdo através de curtidas, comentários e compartilhamento.

Tabela 2. Tabela comparativa do alcance da comunidade nas redes sociais Dados colhidos em 21/03/2021

\begin{tabular}{|l|c|c|c|c|c|}
\hline & Instagram & Facebook & Youtube & LinkedIn & Telegram \\
\hline Número de Seguidores & 1.249 & 715 & 855 & 4.124 & 770 \\
\hline Alcance & 1.330 & 341 & 2.200 & 593 & - \\
\hline Impressões & 12.891 & 74 & 12.000 & 4.100 & - \\
\hline Envolvimento & 657 & 41 & 79 & 757 & - \\
\hline
\end{tabular}

\subsection{Discussões}

Vale ressaltar alguns pontos de grande desenvolvimento da comunidade. Algumas iniciativas e momentos dialogaram mais com as participantes. Tais momentos devem ser discutidos e comparados para aumentar o impacto.

Realizar os eventos de forma on-line foi muito benéfico, pois inicialmente havia uma centralização em São Paulo, por haver muitas organizadoras que moravam nesta região. Com isso, mulheres de outras regiões do país começaram a participar dos eventos e submeter palestras. Atualmente há 14 estados e DF (São Paulo, Rio de Janeiro, Rio Grande do Sul, Minas Gerais, Pernambuco, Santa Catarina, Pernambuco, Ceará, Maranhão, Alagoas, Amazonas, Distrito Federal, Goiás, Pará e Bahia) de acordo com a pesquisa realizada em março de 2021 pelo Telegram. Além disso, as mais de 35 horas de conteúdo produzido nas lives pelo Youtube ficam disponíveis após o evento. 
Outra ideia foi de criar eventos temáticos, nos quais, durante um mês inteiro, todas as lives são sobre aquele determinado tópico. Percebeu-se também a necessidade de explorar mais conteúdos para mulheres que estavam mudando de área e começando agora em IA. Foi observado que grande parte das pessoas que participam têm diversas áreas de formação e não apenas tecnologia, tais como: física, letras, administração, jornalismo e engenharia química.

Um ponto também relevante percebido pelas integrantes da comunidade foi a falta de representatividade feminina nos eventos de tecnologia. Muitas sentiam-se isoladas e não confortáveis de participar. Portanto, pensando nisso, foram realizados vários sorteios para ingressos de alguns eventos de tecnologia do Brasil, em especial o The Developers Conference, seja por meio da doação das próprias integrantes da comunidade ou de empresas parceiras. Isso gerou muito engajamento nas redes sociais, sendo algo que as participantes gostaram.

Além disso, é possível também tecer comparações entre o que está sendo realizado na AI Girls e em outros projetos que compartilharam suas experiências na literatura. A comunidade Minas da TI [Vieira et al. . 2017] conta somente com palestrantes femininas, assim como a AI Girls, mas todos os seus eventos são restritos a mulheres. Também, diferente do apontado por Souza et al. (2017) no projeto Tech Ladies, na AI Girls não há embaixadoras e as organizadoras não são fixas. Decidiu-se por isso pois acredita-se que o papel de apoiar e ser referência como embaixadora pode ser dado a todas as integrantes, gerando um sentimento de pertencimento, e periodicamente são abertas inscrições para novas organizadoras, palestrantes e mentoras da comunidade nas redes sociais, caso as mulheres desejem ter um papel mais ativo.

\subsection{Estatísticas sobre Evento de Aniversário da Comunidade}

Ao completar um ano, a comunidade organizou um evento on-line através do YouTube, totalmente gratuito com duração de 3 dias, sendo cada dia de um assunto específico. Foi o maior evento realizado pela comunidade desde a sua criação, por isso, julgou-se interessante relatar os resultados aqui de maneira mais específica.

As palestrantes foram majoritariamente captadas através de formulário Call4Papers e dividas entre as trilhas de carreira, dados e IA. Algumas palestrantes foram representando parceiros da comunidades, os quais também forneceram brindes como ebook, curso de formação de ciência de dados da Alura e vale da saraiva e licença de 4 meses do Coursera pela Raízen.

O evento teve um total de 650 pessoas inscritas de 8 estados brasileiros, sendo $80 \%$ mulheres $(n=520)$ e $42 \%$ delas procurando ingressar na área de IA e Ciência de Dados $(n=219)$.

Foram extraídas informações sobre as visualizações do conteúdo a cada dia do evento (Tabela 3). No primeiro dia, o evento focou em carreira e representatividade tendo 4 horas e 16 minutos de duração . O principal objetivo era de auxiliar as mulheres que estão em transição ou no início da carreira. De acordo com um report extraído do Youtube Studio e visualizado na tabela abaixo, consegue-se observar que a maior parte do público assistiu a e interagiu mais na trilha de carreira e representatividade. No segundo dia, foi a área de ciência de dados, tendo 4 horas e 47 minutos de duração. O principal objetivo foi de auxiliar as mulheres a entender a importância e como trabalhar com os dados. No 
terceiro dia, o evento focou na área de IA tendo 4 horas e 52 minutos de duração. O principal objetivo era de mostrar como desenvolver sistemas de Inteligência Artificial na prática e como isso é usado em pesquisas e nas empresas.

Levantou-se a hipótese que as mulheres gostariam de assistir temas voltados à carreira nas áreas de IA e ciência de dados. Isso pôde ser comprovado com dados anteriores dos vídeos que mais têm visualizações no canal do Youtube da comunidade. Eles são voltados ao tópico de carreira, como por exemplo a live "Como consegui me tornar uma cientista de dados sem ter formação em tecnologia?"com a Fernanda Santos, que teve mais de mil visualizações e mais de 96 interações no chat. A partir disso, o primeiro semestre de 2021 será dedicado a mulheres em transição de carreira.

Percebeu-se também que, além de mais visualizações, tal tema teve um percentual maior de mulheres assistindo. Isso pode ser devido a necessidade do público feminino conseguir a inserção no mercado de trabalho na área de TI, seja no início ou mudança de carreira. E também pela necessidade de encontrar outras mulheres para trocas de experiências e auxilio emocional. O homens, por sua vez, tiveram maior participação nos dias com conteúdo, não voltado ao gênero.

Tabela 3. Números do primeiro dia de evento

\begin{tabular}{|l|l|l|l|}
\hline & Primeiro dia & Segundo dia & Terceiro dia \\
\hline Duração do evento & $\begin{array}{l}\text { 4 horas e 16 } \\
\text { minutos }\end{array}$ & $\begin{array}{l}\text { 4 horas e 47 } \\
\text { minutos }\end{array}$ & $\begin{array}{l}\text { 4 horas 52 } \\
\text { minutos }\end{array}$ \\
\hline Média de espectadores simultâneos & 78 & 62 & 39 \\
\hline Pico de espectadores simultâneos & 103 & 84 & 49 \\
\hline Espectadores únicos & 285 & 302 & 197 \\
\hline Interações & 734 & 587 & 356 \\
\hline Visualizações pós evento & 506 & 519 & 384 \\
\hline Quantidade de mulheres & $94 \%$ & $65,4 \%$ & $69,4 \%$ \\
\hline
\end{tabular}

O evento trouxe muitos ganhos em relação a visibilidade da comunidade e a retenção de novas mulheres. Desde o primeiro dia do evento os vídeos alcançaram mais de 7 mil pessoas e o canal teve um acréscimo de 145 inscritos, de acordo com um report extraído do Youtube Studio no dia 28 de fevereiro.

Durante as lives e logo após o evento, as pessoas receberam um link para deixarem feedbacks em relação ao evento e os conteúdos que foram disponibilizados. 41 pessoas responderam e $92 \%$ delas eram mulheres. $61 \%$ das pessoas que responderam o formulário não conheciam e não participaram dos eventos anteriores da comunidade e $100 \%$ delas informaram que participarão dos próximos eventos. Além disso, 100\% das mulheres que participaram e responderam ao formulário informaram que o evento cumpriu as expectativas e ajudou-as a obterem novas habilidades.

A crítica mais citada foi a falta de pausa entre as palestras, seguida pela quantidade de palestras no dia. A sugestão apresentada pelas participantes foi ter mais dias de evento, assim conseguindo ter mais pausa entre as palestras, o que está sendo considerado para edições futuras.

Destacam-se também alguns depoimentos e agradecimentos feitos em postagem 
no LinkedIn pelas participantes: "Adorei participar e poder escutar tantas profissionais incríveis, que, com certeza, contribuíram nessa bagagem de conhecimento e deram inspiração para seguirmos motivadas"e outro "[...] E aquele conforto em ver mulheres espalhadas por todo esse país fazendo pesquisas essenciais, se destacando em empresas e levando conhecimento para outras mulheres. Parabéns AI Girls".

\section{Considerações Finais}

As comunidades de tecnologia femininas promovem um ambiente confortável de aprendizagem e senso de comunidade. Observando os números da pouca representação das mulheres no mercado de tecnologia, esses espaços de empoderamento e desenvolvimento profissional se tornam ainda mais necessários. Na área de Inteligência Artificial, isso não é diferente. Assim, foi criada a comunidade AI Girls, com a missão de promover o protagonismo feminino na área.

As ações da comunidade envolvem a promoção de um espaço ativo para compartilhamento de conteúdo com palestras, workshops, blog e discussões temáticas. São realizados: meetups quinzenais, sempre com mulheres da área palestrando; clube de leitura; mentoria para escrita de artigos científicos; entrevistas através da iniciativa "Café com Rosie"; e também sorteio para diversos eventos e cursos.

A organização estruturada da comunidade dá suporte ao crescimento e participação democrática das coordenadoras em todas as iniciativas. Atualmente, há mais de 4000 mulheres participando nas redes da comunidade. O crescimento rápido mostra o interesse por IA e efetividade da iniciativa, sendo suportado também por depoimentos que atestam o impacto do conteúdo na vida delas.

Como próximos passos, espera-se atingir um número ainda maior de pessoas, realizar estudos mais aprofundados de entendimento do público para direcionar as iniciativas e aumentar o alcance para outros estados, captando outras coordenadoras. Em relação aos eventos, há foco no desenvolvimento de workshops sobre LinkedIn, ML, Python e também um prático voltado especificamente para mulheres negras.

\section{Agradecimentos}

Agradecemos à Luiza Bizoni, Maria Fernanda Souza, Thâmara Vilela, Bruna Baldini, Thaissa Bueno Sanches e Mine Caxeiro que fizeram/fazem parte da organização da comunidade e a todas as participantes, pois sem elas não teria sido possível a criação deste espaço. Também a todos os parceiros que ajudam a promover os eventos. E obrigada à professora Carla Rodriguez, pela leitura e opinião sobre o artigo.

\section{Referências}

BRASSCOM (2020). Mulheres são minoria no setor de tecnologia. https://brasscom.org.br/mesmo-com-grau-de-instrucao-mais-elevado-mulheressao-minoria-no-setor-de-tecnologia/.

Faceli, K., Lorena, A. C., Gama, J., Carvalho, A., et al.. (2011). Inteligência artificial: Uma abordagem de aprendizado de máquina. Rio de Janeiro: LTC, 2:192.

Ferrazza, D. S. (2020). Meetups como saberes coletivos?: o organizar de espaços alternativos de aprendizagem à luz da teoria baseada em prática. 
Ingram, C. and Drachen, A. (2020). How software practitioners use informal local meetups to share software engineering knowledge. In 2020 IEEE/ACM 42nd International Conference on Software Engineering (ICSE), pages 161-173. IEEE.

Liu, X., He, Q., Tian, Y., Lee, W.-C., McPherson, J., and Han, J. (2012). Event-based social networks: linking the online and offline social worlds. In Proceedings of the 18th ACM SIGKDD international conference on Knowledge discovery and data mining, pages 1032-1040.

Peres, S. B. and Gomes, E. H. (2020). Estudo sobre o empoderamento feminino através de comunidades de tecnologia. In Anais do XIV Women in Information Technology, pages 219-223. SBC.

Sander, T. (2005). E-associations: using technology to connect citizens: the case of meetup. com. American Political Science Association.

Santiago, S. and de Andrade, C. R. (2018). Iniciativas de inserção de mulheres no mercado de tecnologia da informação: Análise das experiências de participantes brasileiros do desafio technovation. In Anais do XII Women in Information Technology. SBC.

Souza, A. C., Perkoski, I., Veiga, K., and Romankiv, V. (2017). Relato tech ladies: redes de colaboração entre mulheres na tecnologia. In Anais do XI Women in Information Technology. SBC.

Stathoulopoulos, K. and Mateos-Garcia, J. C. (2019). Gender diversity in ai research. Available at SSRN 3428240.

Vieira, C. C., Soares, A. C. R., and Ribeiro, S. F. (2017). Incentivos à igualdade de gênero: estudo de caso de uma comunidade formada somente por mulheres da área de tecnologia. In Anais do XI Women in Information Technology. SBC.

World Economic Forum (2018). The Global Gender Gap Report 2018. http://www3.weforum.org/docs/WEF_GGGR_2018.pdf.

World Economic Forum (2020). The Global Gender Gap Report 2020. http://www3.weforum.org/docs/WEF_GGGR_2020.pdf. 